\title{
Prevalence and determinants of violence against health care in the metropolitan city of Peshawar: a cross sectional study
}

\author{
Muhammad Naseem Khan ${ }^{1,2^{*}}$ (D), Zia UI Haq ${ }^{1}$, Mirwais Khan ${ }^{3}$, Sadia Wali', Faryal Baddia ${ }^{3}$, Shaista Rasul ${ }^{1}$,
} Salman Khan ${ }^{3}$, Maciej Polkowski ${ }^{3}$ and Jessica Yohana Ramirez-Mendoza ${ }^{3}$

\begin{abstract}
Background: Violence against healthcare personnel is a major public health problem. Healthcare personnel are at the frontline dealing with people in stressful and unpredictable situations. Therefore, this study was conducted to determine the prevalence and associated factors of violence against health care personnel.

Methods: A cross sectional study was conducted in the district Peshawar. Healthcare personnel from public and private sectors working in both the primary and tertiary levels of healthcare were invited to participate. Violence was assessed through a structured questionnaire previously used in Pakistan and was defined as experiencing and/ or witnessing any form of violence in the last 12 months. Mental health was assessed through the General Health Questionnaire. Logistic regression was used to estimate the association of violence against healthcare personnel with psychological distress and demographic characteristics. Data entry and analysis were conducted in STATA 14.

Results: A total of 842 healthcare personnel participated in the study. The prevalence of violence experienced and/ or witnessed by healthcare personnel in Peshawar was 51\%. Verbal violence remained the predominant form of violence and almost half of the healthcare personnel (45\%) were exposed to it. A quarter of the respondents (24\%) reported physical violence alone or in combination with other forms of violence. In almost two third of the incidents the perpetrators were either attendants, relatives or the patients. The emergency unit and wards within healthcare facilities were the most common places where violent events took place. The major factors responsible for the violent incidents were communication failure, unreasonable expectations and perceived substandard care. No uniform policy/procedure existed to manage the incidents and the healthcare personnel adopted different responses in the wake of violent events targeting health care. Working in public healthcare facilities and having a larger number of co-workers/colleagues significantly increased the risk of violence in the healthcare settings while being a paramedic significantly reduced the risk as compared to physicians.

(Continued on next page)
\end{abstract}

\footnotetext{
* Correspondence: drnasim@kmu.edu.pk

This study was conducted under the framework of the Health Care in

Danger (HCiD) initiative of the International Committee of the Red Cross

(ICRC).

'Khyber Medical University, Institute of Public Health \& Social Sciences

(IPH\&SS), Phase V, Hayatabad, Peshawar, Pakistan

2Department of Psychological Medicine, University of Liverpool, Liverpool, UK

Full list of author information is available at the end of the article
}

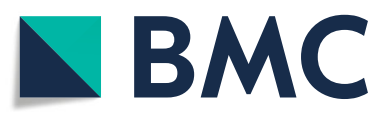

(c) The Author(s). 2021 Open Access This article is licensed under a Creative Commons Attribution 4.0 International License, which permits use, sharing, adaptation, distribution and reproduction in any medium or format, as long as you give appropriate credit to the original author(s) and the source, provide a link to the Creative Commons licence, and indicate if changes were made. The images or other third party material in this article are included in the article's Creative Commons licence, unless indicated otherwise in a credit line to the material. If material is not included in the article's Creative Commons licence and your intended use is not permitted by statutory regulation or exceeds the permitted use, you will need to obtain permission directly from the copyright holder. To view a copy of this licence, visit http://creativecommons.org/licenses/by/4.0/. The Creative Commons Public Domain Dedication waiver (http://creativecommons.org/publicdomain/zero/1.0/) applies to the data made available in this article, unless otherwise stated in a credit line to the data. 
(Continued from previous page)

Conclusions: Violence against healthcare personnel is a serious public health issue and the prevalence is quite high. A holistic effort is needed by all stakeholders including healthcare community, the administration, lawmakers, law enforcement, civil society, and international organizations.

Keywords: Healthcare personnel, Physical violence, Verbal violence, Pakistan, Peshawar

\section{Background}

The World Health Organization (WHO) defines violence as "the intentional use of physical force or power, threatened or actual, against oneself, another person, or against a group or community that either results in or has a high likelihood of resulting in injury, death, psychological harm, mal-development, or deprivation" $[1,2]$. Similarly the World Health Organization defines workplace violence as "incidents where staff are abused, threatened or assaulted in circumstances related to their work, including commuting to and from work, involving an explicit or implicit challenge to their safety, wellbeing or health" [3]. Healthcare personnel are ranked as one of the most exposed group experiencing violence and aggressive behavior. According to the International Labor Office (ILO), workplace violence affects all of the sectors and workers, however health sector is the most highly affected sector [4]. Therefore, violence against healthcare personnel is a major public health problem. Workplace violence can be physical, sexual or psychological in nature and can be actual or threatened [3].

Violence against healthcare personnel is a global phenomenon and studies have been conducted to report the prevalence and associated factors. Studies have reported violence in the Western World [5, 6], Middle East [7] and sub-Saharan Africa [8]. In Pakistan, violence against healthcare personnel is not a new area and has been studied previously. A nationwide study in the major tertiary care hospitals of Pakistan reported more than $70 \%$ healthcare personnel in the emergency departments having experienced some form of violence in the 2 months preceding the study. While physical abuse was faced by $12 \%$ physicians, verbal abuse was experienced by $65 \%$ [9]. The study found that male physicians were more likely to be victims of violence. Similar findings were reported by another study in Karachi, where one in six and three in five physicians reported physical or verbal abuse, respectively, in the past 12 months [10]. In another research study on violence against healthcare almost two thirds of the participants had either experienced or witnessed some kind of violence in the 1 year preceding the study. The main reasons for violence included unreasonable expectations, communication failure, human error, unexpected outcomes, and perception of substandard care [11]. Likewise, emergency department and wards were the most common sites of violence. Due to the front line nature of the work, violence in the emergency department is more prevalent than in other areas of healthcare facilities [12]. Lack of preventive policies, educational inadequacy, unwillingness to report assaults given that violence is considered as part of the job by the healthcare personnel, and unmet expectations of patients and their family are some of the major reasons for violence [13].

Healthcare personnel face particular risks as they are at the frontline dealing with people in stressful, unpredictable and potentially volatile situations. Research on violence against healthcare has been conducted mostly in the southern part of the country mainly Karachi, with no local research on the prevalence, types, and major reasons of violence against healthcare personnel in district Peshawar. Peshawar is located in the northern part of the country with different language, socioeconomic levels, ethnicities, values and culture; therefore, the objectives of the current study were to determine the prevelance and associated factors of violence against healthcare in district Peshawar and also find out association of this violence with the psychological health of the healthcare personnel. The current study was conducted to address this knowledge gap to enable policy makers and planners to develop evidence-based measures for the prevention and control of violence against healthcare.

\section{Methods}

\section{Study design and settings}

A cross-sectional study design was employed for this research. The study was conducted in district Peshawar from April to November of 2017. Healthcare personnel including physicians, nurses, paramedical staff and supporting staff (ward orderlies, ambulance drivers, and gate keepers) were included in the study. The participants were invited from the three main public sector tertiary care hospitals (total 3 in number in district Peshawar) and a sample of private tertiary care hospitals and primary healthcare facilities. Nine Basic Health Units (BHUs) and three Rural Health Centers (RHCs) were randomly selected from the list of primary healthcare facilities in the district. Similarly from the list of private sector tertiary care hospitals two were randomly selected. 
Sample size calculations were based on the anticipated frequencies of verbal and physical abuse taken from a previous study conducted in Karachi, Pakistan [11]. The sample size was calculated for each of the main strata based on the assumptions of an alpha of 0.05 , confidence interval of $95 \%$ and a prevalence of violence from a previous study in Karachi of $65.9 \%$. The sample size based on these assumptions and the actual number in each category was calculated through OpenEpi version 3.01 [14]. The total sample size for physicians was 293, nurses 284, paramedics 274 and support staff 270 . Assuming a non-response rate of $10 \%$, additional sample was enrolled within each of the four main categories making a total sample size of 1233 . The final sample was proportionally invited from each of the main tertiary care hospitals.

A multistage sampling technique was used. Human resources data from the three-major tertiary care public hospitals was provided by the concerned administration entities and was grouped as physicians, nurses, paramedical staff and support staff. Further stratification was done by segregating the above categories into those working in other departments and those specifically deployed in the Accident and Emergency department. Further stratification was done based on their level of seniority as follows: physicians were stratified into senior physicians (registrar and above) and junior physicians (trainee physicians, medical officers and house officers); nurses were stratified into head nurse and staff nurse; paramedics into senior technicians and junior technicians; and support staff into ambulance drivers, computer operators, ward orderlies and security personnel. For private hospitals and primary healthcare centers, cluster sampling technique was applied. Therefore, at first, a random cluster of centers were selected from all the public and private sector hospitals. In the second stage all the healthcare personnel working in CDs/BHUs were sampled for the study while for RHCs and private hospitals, $50 \%$ of physicians, nurses, paramedics and support staff were randomly selected.

Healthcare personnel with at least 1 year of work experience in the healthcare settings were enrolled in the study. Healthcare personnel with less than a year of professional experience, those who were on sick/maternity leave, and those who retired during the study period or were transferred at the time of data collection were excluded.

\section{Measures}

\section{Violence against healthcare personnel}

Violence was assessed through a modified instrument from the Joint Programme on Workplace Violence in the Health Sector of the International Labour Office, the International Council of Nurses, the World Health
Organization, and the Public Services International [15]. This instrument has been translated to the local language (Urdu) and used in similar studies conducted in Karachi $[10,11]$. For this study, violence was defined as any individual or group aggressive behavior or exercise of power, which is socially non-acceptable, turbulent, and often destructive. It was mainly assessed whether the respondent had experienced or witnessed any form of violence in the last 12 months. Physical violence was defined as the use of "physical force against another person that results in physical, sexual, or psychological harm and includes beating, kicking, slapping, stabbing, shooting, pushing, biting and pinching, among others". Verbal abuse included "bullying, mobbing, harassment, and verbal abuse that humiliates, degrades or otherwise indicates a lack of respect for the dignity and worth of an individual". Information gathered from the participants of the study included demographics of the healthcare personnel and administrative information of the healthcare institution. Specific workplace violence questions allowed to assess how worried the healthcare personnel was regarding violence in the healthcare settings; which was rated on a scale from not at all to very worried. Furthermore, details on specific incidents of violence were collected to assess whether violence was physical or verbal, the incident frequency, the type of perpetrators, the location where violence took place within the healthcare institution, and the time of the incident. Healthcare personnel provided information about the main reasons behind the incident, the response following the incident of violence by the healthcare personnel and their recommendations to reduce such incidents or mitigate the impact of such incidents in the future.

\section{Psychological distress}

Psychological distress was measured through the General Health Questionnaire (GHQ-12) [16]. It's score ranges from 0 to 36 and were defined as follows: score < 12 were coded as normal, $12-20$ as distress and more than 20 as severe distress. GHQ-12 has been used and validated in Pakistan with around $90 \%$ sensitivity and specificity [17].

\section{Data collection and analysis}

Before actual data collection, a team of research assistants with experience in data collection were trained on the data collection instruments. Data were collected daily from the research assistants at KMU by the research coordinator. The research coordinator handed over the data files to data entry team for entry/cleaning of data. Any discrepancy and missing data were reported back immediately to the research assistants for clarification. Data entry was done in STATA 14. Mean and 
standard deviation measures was used for descriptive statistics of scale data, and frequencies and percentages for categorical data. For association of violence against healthcare personnel and psychological distress and other demographic factors chi square was used for univariate analysis and logistic regression for multivariate analysis. Associations were considered significant at $p$ value of $<0.05$.

\section{Ethical approval}

The research proposal was reviewed by the institutional and university level boards and committees, along with the approval from the hospital administration. Ethical approval was obtained from the Ethics Board of Khyber Medical University, Peshawar. Informed written consent was obtained from every participant after explaining the purpose of research and providing written information to participants. The right to withdraw from the study at any time without providing a reason was reinforced to all participants during consent and prior to the conduct of the survey.

\section{Results}

A total of 1283 healthcare personnel were approached for participation in the current study. Of them 187 were part of the exclusion criteria of having less than 1 year of professional experience, were transferred, retired or on leave and as such were excluded from the study. An additional 204 participants refused participation. Lastly, 50 forms were incompletely filled and hence were excluded from analysis. Therefore, analysis was done on a total of 842 healthcare workers.

\section{Demographic and job characteristics}

Table 1 below shows the demographic and job characteristics of the participants and their associations with exposure to violence in the last 1 year. Statistically significant associations were observed between violence and number of co-workers, public healthcare facility, and job categories of the healthcare personnel. Healthcare personnel exposed to violence had significantly higher levels of preoccupation about workplace violence with $24.6 \%$ of them feeling worried and $17.8 \%$ extremely worried; while among the healthcare personnel not exposed to violence, only $2.7 \%$ felt worried and $2.9 \%$ extremely worried. The current study found that age, work experience, gender, marital and distress status of healthcare personnel, and level of the healthcare facility (primary or tertiary) had no association with exposure to violence.

\section{Prevalence \& pattern of violence}

The prevalence and patterns of different types of violence against health care (verbal, physical and damage to facility) is presented in Table 2. There was exposure to violence among $51 \%$ of the sampled healthcare workers in Peshawar district over a period of 1 year. More than a quarter of the respondents (26\%) had experienced as well as witnessed violence against health care. Among respondents who had witnessed and/or experienced violence, verbal violence remained the most prevalent form (45\%), followed by a combination of physical and verbal abuse (23\%) and a combination of physical, verbal and facility damage (22\%). A higher prevalence of violence was observed in public healthcare facilities, and among physicians and support staff compared to nurses and paramedics.

Table 3 shows the characteristics of the violent incidents. In more than two thirds of the incidents the perpetrator was either the attendant/relative of the patient $(44 \%)$ or the patient $(20 \%)$. In $85 \%$ of incidents there was involvement of two or more perpetrators in each event. Emergency departments (34\%) and wards (30\%) were the common sites of violent incidents in the healthcare facilities, and more than half (52\%) of the incidents took place during the morning shift.

\section{Mental health effects of violence}

The mental health effects on healthcare personnel who had witnessed and /or experienced violence are summarized in Fig. 1 below. Around two-thirds of the participants exposed to violence had experienced some form of mental health consequences following the incidents. Regarding the GHQ 12 status, half of the respondents had a score of less than 12, $45 \%$ were distressed with a score of 12-20, while the remaining $5 \%$ were severely distressed with a score of more than 20 .

\section{Factors responsible and events following the violent incidents}

Table 4 summarizes the factors responsible for the development of violence against healthcare and the actions taken by the victims following the events. The table also shows the factors that the respondents of the study deemed as generally contributing to the development of violence against healthcare and the recommendations made by the participants for the prevention of violence in healthcare settings. Communication failure (71\%), unreasonable expectations (61\%), perceived substandard care (55\%), management failure (55\%) and human error (51\%) were the predominant factors responsible for the violent incidents. Other important factors included financial pressure, facility failure, a smaller number of staff and unexpected outcomes. Following the incident around two thirds (61\%) defended themselves and around half (52\%) told the attacker to stop, the victim sought 
Table 1 Demographic \& Job Characteristics and association with Violence

\begin{tabular}{|c|c|c|c|}
\hline Variables & $\begin{array}{l}\text { Exposed to violence } \\
N=427 \\
\mathrm{n}(\%)\end{array}$ & $\begin{array}{l}\text { Not exposed to violence } \\
N=415 \\
\mathrm{n}(\%)\end{array}$ & Significance \\
\hline Age in years (Mean $\pm S D$ ) & $35.44 \pm 9.2$ & $35.52 \pm 10.0$ & 0.910 \\
\hline Work Experience in years (Mean \pm SD) & $9.85 \pm 8.88$ & $9.85 \pm 8.67$ & 0.868 \\
\hline No of co-workers (Mean \pm SD) & $7.14 \pm 7.22$ & $4.90 \pm 4.68$ & $<0.001$ \\
\hline \multicolumn{4}{|l|}{ Gender } \\
\hline Male & $296(69.32)$ & $278(66.99)$ & \multirow[t]{2}{*}{0.468} \\
\hline Female & $131(30.68)$ & $137(33.01)$ & \\
\hline \multicolumn{4}{|l|}{ Level of healthcare facility } \\
\hline Primary & $37(8.7)$ & $38(9.2)$ & \multirow[t]{2}{*}{0.802} \\
\hline Tertiary & $390(91.3)$ & $377(90.8)$ & \\
\hline \multicolumn{4}{|l|}{ Type of Health facility } \\
\hline Public & $396(92.7)$ & $298(71.8)$ & \multirow[t]{2}{*}{$<0.001$} \\
\hline Private & $31(7.3)$ & $117(28.2)$ & \\
\hline \multicolumn{4}{|l|}{ Marital status } \\
\hline Single & $116(27.2)$ & $123(29.6)$ & \multirow[t]{2}{*}{0.426} \\
\hline Married & $311(72.8)$ & $292(70.4)$ & \\
\hline \multicolumn{4}{|l|}{ Job category } \\
\hline Physicians & $104(24.4)$ & $68(16.4)$ & \multirow[t]{4}{*}{$<0.001$} \\
\hline Nurse & $84(19.7)$ & $109(26.3)$ & \\
\hline Paramedics & $89(20.8)$ & $126(30.4)$ & \\
\hline Support Staff & $150(35.1)$ & $112(27.0)$ & \\
\hline \multicolumn{4}{|c|}{ How worried are you regarding violence in your work settings } \\
\hline Not worried & $106(24.8)$ & $226(54.5)$ & \multirow[t]{5}{*}{$<0.001$} \\
\hline Somewhat worried & $57(13.4)$ & $92(22.2)$ & \\
\hline Little worried & $83(19.4)$ & $74(17.8)$ & \\
\hline Worried & $105(24.6)$ & $11(2.7)$ & \\
\hline Extremely worried & $76(17.8)$ & $12(2.9)$ & \\
\hline \multicolumn{4}{|l|}{ Distress status } \\
\hline Normal & $206(48.2)$ & $214(51.6)$ & \multirow[t]{3}{*}{0.621} \\
\hline Somewhat distress & $200(46.8)$ & $181(43.6)$ & \\
\hline Severe distress & $21(5.0)$ & $20(4.8)$ & \\
\hline
\end{tabular}

counselling (43\%) and tried to report the incident to police (45\%). The healthcare personnel were asked to identify factors generally contributing to violence in healthcare settings. The respondents identified low awareness among the general public, workload of healthcare personnel, lack of facilities, shortage of staff and high expectations as major factors contributing to violence against health care. The participants reported improvement in healthcare services, increase of healthcare personnel, improvement in overall public awareness, controlled entry of attendants, provision of security and abolition of VIP protocols as recommendations for the prevention of violence in the healthcare settings.
Table 5 shows the associations of violence against healthcare personnel through regression analysis with presentation of adjusted and unadjusted odds ratios with 95\% confidence intervals. The likelihood of experiencing and/or witnessing violence was significantly associated with public healthcare facilities, larger number of coworkers/colleagues, worry regarding violence in the healthcare settings and job category. Working in public healthcare facilities, worry regarding violence in the healthcare settings and having a larger number of coworkers/colleagues significantly increased the odds of a healthcare personnel experiencing and/or witnessing violence while being a paramedic significantly reduced the odds as compared to physicians. 
Table 2 Prevalence \& Pattern of violence

\begin{tabular}{ll}
\hline Prevalence of Violence & $\begin{array}{l}\boldsymbol{N}=\mathbf{4 2 7} \\
\mathbf{n}(\%)\end{array}$ \\
\hline Experienced and/or Witnessed & $427(51)$ \\
Experienced only & $33(4)$ \\
Witnessed only & $176(21)$ \\
Experienced and Witnessed & $218(26)$ \\
Type of healthcare facility & \\
Public & $396(93)$ \\
Private & $31(7)$ \\
Level of healthcare facility & \\
Primary healthcare facility & $37(9)$ \\
Tertiary healthcare facility & $390(91)$ \\
HealthCare Personnel & $104(24)$ \\
Physicians & $84(20)$ \\
Nurses & $89(21)$ \\
Paramedics & $150(35)$ \\
Support Staff & \\
Gender of the healthcare personnel & $296(69)$ \\
Male & $131(31)$ \\
Female & $35(8)$ \\
\hline Vattern of violence & \\
Verbal violence only & $100(23)$ \\
Verbal, Facility damage \& Physical violence & \\
Only Facility damage & $(22)$ \\
Physical \& Facility damage & $(0.7)$ \\
\hline
\end{tabular}

\section{Discussion}

The current study was the first of its kind to determine the prevalence of violence against healthcare personnel and the associated factors through a large representative sample of both the public and private healthcare sectors, including primary and tertiary levels of care, in district Peshawar, Pakistan. The prevalence of violence witnessed and/or experienced by healthcare personnel in Peshawar was $51 \%$. Verbal violence remained the predominant form of violence witnessed and/or experienced. Almost half of the healthcare personnel (45\%) involved in the study had witnessed and/or experienced verbal violence. Furthermore, a quarter of the respondents (24\%) reported witnessing and/or experiencing physical violence alone or in combination with other forms of violence. In almost two third of the incidents the perpetrators were either attendants, relatives of patients or the patients. The emergency unit and wards within healthcare facilities were the most common places where violent events took place. The
Table 3 Characteristics of the violent incidents

\begin{tabular}{ll}
\hline Characteristics of violent incidents & Percentages \\
\hline Perpetrators of violence & 44 \\
Attendant/relative & 20 \\
Patient & 14 \\
General public & 8 \\
VIP escort & 7 \\
Staff member & 5 \\
Security personnel & 2 \\
Others & \\
Number of perpetrators involved & 15 \\
One & 72 \\
Two-five & 13 \\
More than five &
\end{tabular}

Number of violent incidents experienced and/or witnessed over 12 months

$\begin{array}{ll}\text { Once } & 12 \\ \text { Two-five times } & 55 \\ \text { More than five } & 33\end{array}$

Number of victims affected in violent incidents

$\begin{array}{ll}\text { One } & 40 \\ \text { Two-five } & 55 \\ \text { More than five } & 5\end{array}$

Place of violence within healthcare facility

$\begin{array}{ll}\text { Emergency room } & 34 \\ \text { Ward } & 30 \\ \text { Hospital parking area } & 8 \\ \text { Radiology } & 8 \\ \text { Intensive Care Unit } & 5 \\ \text { Others } & 15\end{array}$

Distribution of the violent events by time

$\begin{array}{ll}\text { Morning shift (8 to 2) } & 52 \\ \text { Evening (2-8) } & 20 \\ \text { Night (8) } & 13 \\ \text { Do not remember } & 15\end{array}$

\section{Consequences of violence}

Injured victims requiring treatments

75

major factors responsible for the violent incidents were communication failure, unreasonable expectations and perceived substandard care. No uniform policy/procedure existed to manage the incidents and the healthcare personnel adopted different responses in the wake of violent events targeting healthcare. Working in public healthcare facilities and having a larger number of co-workers/ colleagues significantly increased the risk of violence in the healthcare settings while being a paramedic significantly reduced the risk as compared to physicians. 


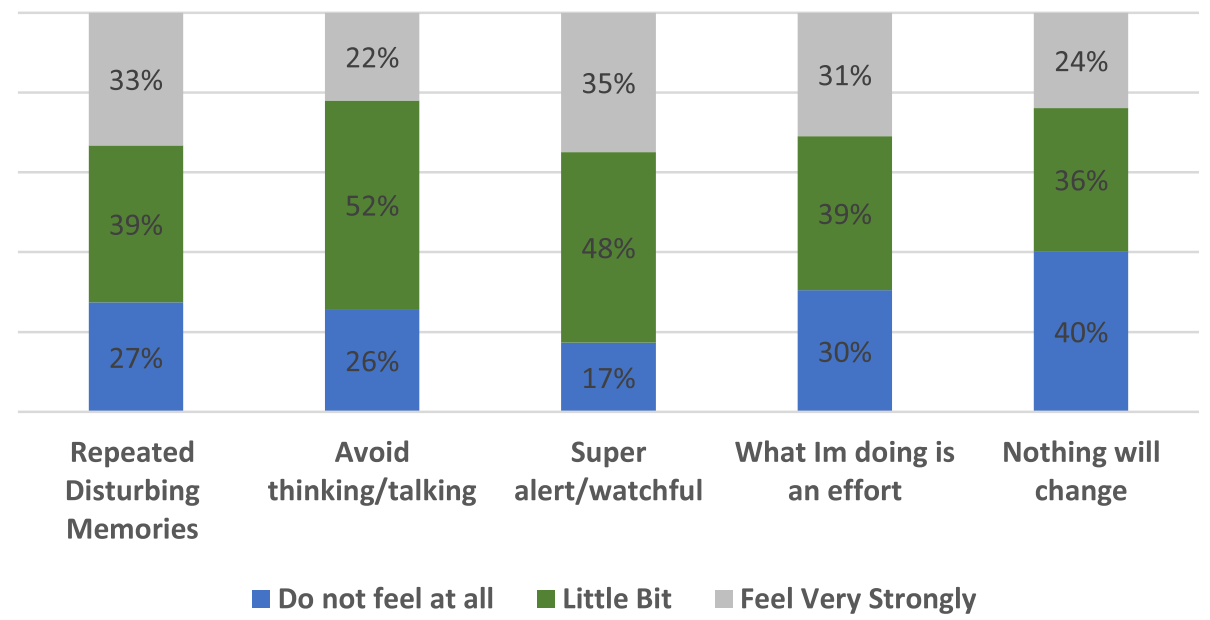

Fig. 1 Mental health effects of the violence incident

The main strength of the current study was that the sample was drawn from the official records and healthcare workers were invited randomly, reflecting the true prevalence of violence against healthcare. Furthermore, healthcare workers were invited from public/private and primary/tertiary level of care. The main limitation of the study was that due to the cross-sectional nature, the temporal relationship between exposure and outcome could not be established. Therefore, a study design exploring the temporal relationship between factors and violence against health care should be employed along with patient/attendant perspective in future studies. Likewise 204 participants refused participation and no baseline information collected to compare with the respondents. This is another limitation as the nonrespondents might have been more exposed to violence against healthcare.

Violence against healthcare is not a new phenomenon as healthcare professionals around the world are exposed to some form of violence $[1,9,10$, $18,19]$. The current study portrays a similar picture and reported verbal violence as the most common form of violence compared to physical or other forms $[18,20]$. Likewise, physical violence is experienced by $(8-38 \%)$ of health workers worldwide [1]. The frequency of experiencing/witnessing physical abuse (24\%) as per the findings of the study are consistent with this range. However, this figure is considerably higher when compared with the country wise frequency of physical abuse reported by healthcare workers in Bulgaria (7.5\%), Brazil (6.4\%), Lebanon (5.8\%), Thailand (10.5\%), South Africa (9-17\%) and Karachi, Pakistan (16\%) [4, 10, 21]. These different results could be explained by the different recall times in these studies as the shorter period will have better recall, but few incidents compared to the longer period.

The higher rates of physical violence could also be due to the large number of relatives/attendants accompanying the patients in this part of the world and is considered a culutural norm to visit patients even in the hospital. This at time is required to facilitate the management of the patient in the hospital as not all the services and care (laboratory tests/medicines and nursing care) is provided by the hospital staff. In the current study more than one perpetrator was involved in $85 \%$ of the incidents of violence. Multiple studies conducted across various cities of Pakistan have revealed that the main perpetrators of violence against healthcare workers were attendants of patients $[9,19]$. Therefore, policy should be adopted to limit the entry of relatives/attendants with the patients and raise awareness among the general community on the attendant's restriction. The shape and impact of implementation of this policy should be guided by continuous evaluation to remain mindful of the cultural needs and the duty to ensure safety of patients and healthcare personnel.

Consistent with the findings of other studies, our results show that amongst the various cadres of healthcare workers, doctors encounter fewer incidents of violence whereas paramedics experienced the greatest number of violent incidents. Communication failure (71\%), unreasonable expectations (61\%), management failure (55\%) and perceived substandard care (55\%) were reported as the primary causes of violence against healthcare workers. Communication failure was the biggest reason reported in the current study. This was reported by the healthcare personnel in a study where they believed that 
Table 4 Factors responsible and events following the violent incidents

\begin{tabular}{|c|c|}
\hline Characteristics of the violence incident & n (\%) \\
\hline \multicolumn{2}{|l|}{$\begin{array}{l}\text { Factors responsible for the violent incidents } \\
\text { (multiple responses) }\end{array}$} \\
\hline Communication failure & $302(71)$ \\
\hline Unreasonable expectation & $261(61)$ \\
\hline Management failure & $234(55)$ \\
\hline Perceived substandard care & $233(55)$ \\
\hline Human error & $219(51)$ \\
\hline Financial pressure & $195(46)$ \\
\hline Less number of healthcare personnel & $147(34)$ \\
\hline Unexpected outcome & $128(30)$ \\
\hline
\end{tabular}

Responses of the victims following the violent incident (multiple responses)

Tried to pretend it never happened

Attacker was told to stop

The victim defended him/herself

Sought counseling

Sought help from association

Reported to Police

Reasons for not taking any action (multiple responses)

It was not important

Felt ashamed

Felt guilty

Useless

Afraid of negative consequences

Did not know who to report to

Factors contributing to violence in health-care settings (multiple responses)

Low awareness in the general public

Workload of healthcare personnel

High expectations

VIP protocols

Lack of facilities at the healthcare facility

Shortage of healthcare personnel

Recommendations for the prevention of violence (multiple responses)

Improvement in healthcare services

Healthcare personnel increase

Provision of security

No VIP protocols

$129(15)$

Controlled entry of attendants

$289(34)$

Overall public awareness

their dismissive and authoritative attitude and a lack of empathy contributes to emergence of incidents of violence [22]. The other factors reported have also been

reflected in similar studies conducted across various countries including Pakistan [23-25]. There is a lack of a robust referral system in Pakistan. The concept of gatekeeping in healthcare does not exist. Patients tend to seek care at tertiary level facilities even for minor selflimiting ailments. This translates into unrealistic expectations by the patients and puts a toll on the already compromised facilities. Furthermore, it is evident from the findings of this study, the health care workers working in public sector facilities experience violence more frequently as compared to those working in the private sector. Public sector hospitals in Pakistan are overburdened. Healthcare workers working at public hospitals cater to an overwhelmingly large number of patients visiting the facilities at all hours of the day. Under these circumstances the healthcare workers have to rush through the patients and are not being able to give the due time and consideration which every patient deserves, thereby resulting in communication failure, management failure and perceived substandard care. Increasing the capacity of workforce and expansion of the facilities has been proposed by the study participants as one of the strategies to overcome these issues. However, one interesting finding was the association of violence with large number of co-workers/colleagues. This might be because the health workers felt confident with the support and resort to violence easily in such settings. Therefore, expansion of the facilities/recruitment of the staff to deal with the workload should be done with additional training on better communication skills, conflict resolution and de-escalation of the violent events.

Another important factor for violence against healthcare is financial pressure (46\%) on part of the patients. This has been reported for violence against healthcare in other low and middle-income countries [26]. In Pakistan $29 \%$ of the population lives below the national poverty line. Despite this staggering figure, the out of pocket health expenditure approximates $70 \%$ of the country's net expenditure on health [27]. Recently the government of Khyber Pakhtunkhwa has initiated social health protection schemes (insurance schemes for admitted patients) covering nearly 49 million people living below the poverty line, for all secondary healthcare admission [27, 28]. These schemes could play a big role in reducing the burden of violence against healthcare.

In more than half $(61 \%)$ of the incidents; the healthcare worker defended himself/herself. Similarly, around half of the healthcare workers verbally told the attacker to stop. One explanation could be that there is no formal system for redressal of these issues at the institutional level. Therefore the healthcare worker has no other choice but to defend him/herself, and the local culture encourages self defense in situation like these. About $45 \%$ of the victims sought counselling and/or 
Table 5 Adjusted and unadjusted Odds ratio of Violence in the healthcare settings

\begin{tabular}{|c|c|c|c|c|}
\hline Variables & Unadjusted Odds ratio $(95 \% \mathrm{Cl})$ & $P$ value & Adjusted Odds ratio $(95 \% \mathrm{Cl})$ & $P$ value \\
\hline Age in years & $0.10(0.99,1.01)$ & 0.910 & $0.98(0.96,1.01)$ & 0.256 \\
\hline Work Experience in years & $1.00(0.99,1.02)$ & 0.868 & $1.00(0.96,1.03)$ & 0.826 \\
\hline No of co-workers & $1.07(1.04,1.09)$ & $<0.001$ & $1.06(1.03,1.09)$ & $<0.001$ \\
\hline Male & $1.11(0.83,1.49)$ & 0.468 & $1.03(0.64,1.64)$ & 0.903 \\
\hline Tertiary healthcare facility & $1.06(0.66,1.71)$ & 0.802 & $0.71(0.40,1.25)$ & 0.233 \\
\hline Public sector health facilities & $5.02(3.28,7.66)$ & $<0.001$ & $3.82(2.35,6.19)$ & $<0.001$ \\
\hline Married & $1.13(0.84,1.52)$ & 0.426 & $1.15(0.76,1.73)$ & 0.503 \\
\hline \multicolumn{5}{|l|}{ Job category } \\
\hline Physicians & Reference category & & & \\
\hline Nurse & $0.50(0.33,0.77)$ & 0.001 & $0.82(0.45,1.50)$ & 0.518 \\
\hline Paramedics & $0.46(0.31,0.70)$ & $<0.001$ & $0.59(0.36,0.97)$ & 0.038 \\
\hline Support Staff & $0.88(0.59,1.30)$ & 0.006 & $1.55(0.90,2.68)$ & 0.115 \\
\hline \multicolumn{5}{|c|}{ How worried are you regarding violence in your work setting } \\
\hline Not worried & Reference category & & & \\
\hline Somewhat worried & $1.32(0.88,1.98)$ & 0.176 & $1.39(0.89,2.17)$ & 0.149 \\
\hline Little worried & $2.39(1.62,3.53)$ & $<0.001$ & $2.85(1.83,4.42)$ & $<0.001$ \\
\hline Worried & $20.35(10.49,39.48)$ & $<0.001$ & $17.91(8.94,35.87)$ & $<0.001$ \\
\hline Extremely worried & $13.50(7.04,25.89)$ & $<0.001$ & $14.10(7.10,28.02)$ & $<0.001$ \\
\hline \multicolumn{5}{|l|}{ Distress status } \\
\hline Normal & Reference category & & & \\
\hline Somewhat distress & $1.15(0.87,1.52)$ & 0.330 & $0.70(0.48,1.00)$ & 0.051 \\
\hline Severe distress & $1.09(0.57,2.07)$ & 0.791 & $0.57(0.25,1.28)$ & 0.174 \\
\hline
\end{tabular}

pursued prosecution whereas $32 \%$ pretended that the abuse did not take place at all. The reasons behind the reluctance of healthcare workers in reporting such incidents of violence are manifold. More than half of the healthcare personnel who did not report the incident deemed it not important or futile. Whereas others either felt threatened by the adverse consequences of seeking help and prosecution or felt that reporting such incidents of violence threatened their self-respect and dignity. Therefore, pretending that the incident did not take place at all seemed like the most suitable reaction in such situations for many. These findings are in conformity with similar studies which reported that amongst the reactions to violence against healthcare, feeling angry, helpless and humiliated are the most commonly reported reactions $[29,30]$. Why is it that health professionals, who are amongst the most revered members of the society find themselves helpless in such situations? One of the reasons could be a lack of trust in the relevant institutions, in the system of accountability and in the formal process of seeking prosecution which in most cases appears like a time consuming and futile exercise. Another reason, as reported by an Iranian study is the lack of responsibility by the health system for redressal in such cases [31]. One of the major reason for underreporting of incidents of violence by health professionals according to a study conducted in Pakistan was the fear of having adverse effect on the victims job and personal life [32]. Clearly this reflects a lack of trust in the management of incidents of violence by the healthcare organizations. Similarly based upon the factors that lead to incidents of violence, another study conducted in Karachi predicted a 5\% reduction in such cases of violence against health professionals by introducing grievance-redressal policies for the staff [33].

Incidents of violence adversely affect the mental health of the healthcare workers. Around half of the healthcare workers $(52 \%)$ in the study felt mentally distressed due to violence instigated against them. The psychosocial consequences of violence have been widely reported in various studies. A systematic review on the aftermath of violence against healthcare stated that amongst the various consequences of violence the psychosocial affects such as depression and anxiety are experienced most frequently by the victims [34]. This could have major implications where one third of the healthcare personnel avoid and pretend that the incident didn't took place.

The phenomenon of violence against health care can have devasting public health consequences, especially for fragile, under-resourced healthcare systems. It holds the 
potential to immensely balloon the healthcare needs, diminish the availability of services and render the access to and provision of healthcare services unsafe and inefficient. For an over-stretched and over-burdened healthcare system, the additional burden imposed by humanitarian consequences of violence against health care can tip the scales between the needs and availability of the healthcare services in a direction detrimental to the health outcomes of populations using the healthcare services.

\section{Conclusion}

Violence against healthcare personnel is a serious public health issue and the prevalence is high. This may have effects on their wellbeing and could lead to low job motivation, which in turn can put the healthcare provision at risk in an already compromised healthcare system in a developing country like Pakistan. Therefore safety of the wounded and the sick, healthcare personnel, healthcare facilities and medical vehicles is important for provision of essential services. Some of the strategies for the prevention and control of violence against healthcare could be

- Development and implementation of "zero tolerance policy for violence in healthcare settings", filling gaps in legislation and establishment of incident reporting mechanism by the provincial health department, administration of hospitals, and other monitoring bodies.

- The administration of all public and private healthcare facilities should train healthcare workers on deescalation of violence [35], effective communication and conflict resolution skills, and ethics [36, 37].

- Administration of healthcare facilities, provincial health department and local law enforcement agencies need to implement measures to improve hospital and staff security and strengthen the needed coordination.

- Provincial health department and administration of healthcare facilities should improve facilitation of patients and attendants through clear signposting and information desks, waiting areas, one attendant policy, and improvement of quality of healthcare services.

- Engagement of media, community elders and religious influencers to raise awareness and counter cultural barriers to increased respect for health care.

These should be assessed through research in the local settings with involvement of not just by the healthcare community, but also needs the support and facilitation from government, the administration, lawmakers, law enforcement, civil society, and international organizations.

\section{Abbreviations}

BHU: Basic Health Units; CD: Civil Dispensary; HCiD: Health Care in Danger; GHQ: General Health Questionnaire; ICRC: International Committee of the Red Cross; ILO: International Labor Office; KMU: Khyber Medical University; RHC: Rural Health Center; WHO: World Health Organization

\section{Acknowledgements}

We are thankful to all the study participants for their time and involvement in the study. We are also very thankful to our team of data collectors and healthcare administration. We are extremely thankful to the management of International Committee of the Red Cross, Pakistan for the technical and financial support of the study.

\section{Authors' contributions \\ MNK, ZUH and MK conceived the idea of this research study and participated in its coordination. MNK, ZUH, FB, SW and SK conducted field research activities and were involved in the data collection, cleaning and initial analysis. Further analysis was supported by SR, MK, MP and JYR. All authors contributed towards preparing the first draft as well as editing and reviewing of the subsequent drafts. The authors read and approved the final manuscript. \\ Funding \\ This study was conducted under the framework of the Health Care in Danger ( $\mathrm{HCiD}$ ) initiative of the International Committee of the Red Cross (ICRC). The funding body has no role in the design of the study and collection, analysis, and interpretation of the data and in writing of the manuscript.}

\section{Availability of data and materials}

The raw data available upon reasonable request from the corresponding author.

Ethics approval and consent to participate

The study was approved by the Ethics Board of Khyber Medical University, Peshawar. Informed written consent obtained from study participants following explanation of the study aims and objectives. All procedures were performed with strict ethical standards.

Consent for publication

Not applicable.

Competing interests

The author(s) declare that they have no competing interests.

\section{Author details}

${ }^{1}$ Khyber Medical University, Institute of Public Health \& Social Sciences (IPH\&SS), Phase V, Hayatabad, Peshawar, Pakistan. ${ }^{2}$ Department of Psychological Medicine, University of Liverpool, Liverpool, UK. ${ }^{3}$ International Committee of the Red Cross, Geneva, Switzerland.

Received: 13 May 2020 Accepted: 14 January 2021

Published online: 10 February 2021

References

1. World report on violence and health. Violence and Injury Prevention. [Cited 2020 Mar 14]. Available from: https://www.who.int/violence_injury prevention/violence/world_report/en/

2. Definition and typology of violence. Violence Prevention Alliance. [Cited 2020 Mar 20]. Available from: https://www.who.int/violenceprevention/ approach/definition/en/

3. World Health Organization. Framework guidelines for addressing workplace violence in the health sector. Geneva: International Labour Office; 2002.

4. Di Martino V, Workplace violence in the health sector. Country case studies Brazil, Bulgaria, Lebanon, Portugal, South Africa, Thailand and an additional Australian study Ginebra: Organización Internacional del Trabajo; 2002. p. 3-42.

5. Hartley D, Ridenour M, Craine J, Costa B. Workplace violence prevention for healthcare workers - an online course. Rehab Nurs. 2012;37(4):202-6. 
6. Vorderwülbecke F, Feistle M, Mehring M, Schneider A, Linde K. Aggression and violence against primary care physicians - a nationwide questionnaire survey. Dtsch Ärztebl Int. 2015;112(10):159.

7. Khademloo M, Moonesi FS, Gholizade H. Health care violence and abuse towards nurses in hospitals in north of Iran. Global J Health Sci. 2013;5(4): 211.

8. Seun-Fadipe CT, Akinsulore AA, Oginni OA. Workplace violence and risk for psychiatric morbidity among health workers in a tertiary health care setting in Nigeria: prevalence and correlates. Psychiatry Res. 2019;272:730-6.

9. Mirza NM, Amjad Al, Bhatti ABH, Shaikh KS, Kiani J, Yusuf MM, et al. Violence and abuse faced by junior physicians in the emergency department from patients and their caretakers: a nationwide study from Pakistan. J Emerg Med. 2012;42(6):727-33.

10. Zafar W, Khan UR, Siddiqui SA, Jamali S, Razzak JA. Workplace violence and self-reported psychological health: coping with post-traumatic stress, mental distress, and burnout among physicians working in the emergency departments compared to other specialties in Pakistan. J Emerg Med. 2016: 50(1):167-77.

11. Baig LA, Shaikh S, Polkowski M, Ali SK, Jamali S, Mazharullah L, et al. Violence against health care providers: a mixed-methods study from Karachi, Pakistan. J Emerg Med. 2018;54(4):558-66.

12. Kowalenko T, Cunningham R, Sachs CJ, Gore R, Barata IA, Gates D, et al. Workplace violence in emergency medicine: current knowledge and future directions. J Emerg Med. 2012;43(3):523-31.

13. Baydin A, Erenler AK. Workplace violence in emergency department and its effects on emergency staff. Int J Emerg Ment Health. 2014;16(2):288-90.

14. Dean AG, Sullivan KM, Soe MM. Open Source Epidemiologic Statistics for Public Health. OpenEpi. [cited 2018 Feb 15]. Available from: http://www. openepi.com/Menu/OE_Menu.htm

15. International Labor Office, World Health Organization, International Council of Nurses, Public Services International. Workplace violence in the health sector country case studies research instruments. (Joint Programme on Workplace Violence in the Health Sector).

16. Goldberg DP. User's guide to the general health questionnaire. Windsor; 1988.

17. Minhas FA, Mubbashar $\mathrm{MH}$. Validation of general health questionnaire in a primary care setting of Pakistan. J Coll Physicians Surg Pak. 1996;6:133-6.

18. Alsaleem SA, Alsabaani A, Alamri RS, Hadi RA, Alkhayri MH, Badawi KK, et al. Violence towards healthcare workers: a study conducted in Abha City, Saudi Arabia. J Fam Community Med. 2018;25(3):188.

19. Nayyer-ul-Islam MY-I, Farooq MS. Workplace violence experienced by doctors working in government hospitals of Karachi. J Coll Physicians Surg Pak. 2014;24(9):698-9.

20. Cashmore AW, Indig D, Hampton SE, Hegney DG, Jalaludin BB. Workplace violence in a large correctional health service in New South Wales, Australia: a retrospective review of incident management records. BMC Health Serv Res. 2012;12(1):245

21. Zafar W, Siddiqui E, Ejaz K, Shehzad MU, Khan UR, Jamali S, et al. Health care personnel and workplace violence in the emergency departments of a volatile metropolis: results from Karachi, Pakistan. J Emerg Med. 2013;45(5): 761-72.

22. Shafran-Tikva S, Chinitz D, Stern Z, Feder-Bubis P. Violence against physicians and nurses in a hospital: how does it happen? A mixed-methods study. Isr J Health Policy Res. 2017;6(1):59.

23. Jiao M, Ning N, Li Y, Gao L, Cui Y, Sun H, et al. Workplace violence against nurses in Chinese hospitals: a cross-sectional survey. BMJ Open. 2015;5:3.

24. Du Y, Wang W, Washburn DJ, Lee S, Towne SD, Zhang H, et al. Violence against healthcare workers and other serious responses to medical disputes in China: surveys of patients at 12 public hospitals. BMC Health Serv Res. 2020;20(1):1-10.

25. Albashtawy M. Workplace violence against nurses in emergency departments in J ordan. Int Nurs Rev. 2013;60(4):550-5.

26. Najafi F, Fallahi-Khoshknab M, Ahmadi F, Dalvandi A, Rahgozar M. Antecedents and consequences of workplace violence against nurses: a qualitative study. J Clin Nurs. 2018;27(1-2):e116-28.

27. Khan SA. Pakistan's march towards universal health coverage. J Ayub Med Coll Abbottabad. 2017;29(4):533-4.

28. Sehat Sahulat Program. [cited 2020 Jan 4]. Available from: https://www. pmhealthprogram.gov.pk/about-us/

29. O'Brien-Pallas L, Hayes L, Wang S, Laporte D. Creating work environments that are violence free. World Hosp Health Serv. 2009;45(2):12-8.
30. Samir N, Mohamed R, Moustafa E, Abou SH. Nurses' attitudes and reactions to workplace violence in obstetrics and gynaecology departments in Cairo hospitals. EMHJ-Eastern Mediterranean Health J. 2012;18(3):198-204.

31. Esmaeilpour M, Salsali M, Ahmadi F. Workplace violence against Iranian nurses working in emergency departments. Int Nurs Rev. 2011;58(1):130-7.

32. Baig LA, Ali SK, Shaikh S, Polkowski MM. Multiple dimensions of violence against healthcare providers in Karachi: results from a multicenter study from Karachi. J Pak Med Assoc. 2018;68(8):1157-65.

33. Shaikh S, Baig LA, Hashmi I, Khan M, Jamali S, Khan MN, et al. The magnitude and determinants of violence against healthcare workers in Pakistan. BMJ Glob Health. 2020;5(4):e002112.

34. Lanctôt N, Guay S. The aftermath of workplace violence among healthcare workers: a systematic literature review of the consequences. Aggress Violent Behav. 2014;19(5):492-501.

35. Baig L, Tanzil S, Shaikh S, Hashmi I, Khan MA, Polkowski M. Effectiveness of training on de-escalation of violence and management of aggressive behavior faced by health care providers in a public sector hospital of Karachi. Pakistan J Med Sci. 2018;34(2):294

36. Dean M, Oetzel JG. Physicians' perspectives of managing tensions around dimensions of effective communication in the emergency department. Health Commun. 2014;29(3):257-66.

37. Brophy JT, Keith MM, Hurley M. Assaulted and unheard: violence against healthcare staff. New Solut J Environ Occup Health Policy. 2018;27(4):581606.

\section{Publisher's Note}

Springer Nature remains neutral with regard to jurisdictional claims in published maps and institutional affiliations.

Ready to submit your research? Choose BMC and benefit from:

- fast, convenient online submission

- thorough peer review by experienced researchers in your field

- rapid publication on acceptance

- support for research data, including large and complex data types

- gold Open Access which fosters wider collaboration and increased citations

- maximum visibility for your research: over $100 \mathrm{M}$ website views per year

At BMC, research is always in progress.

Learn more biomedcentral.com/submissions 